\title{
Drift perturbation's influence on traveling wave speed in KPP-Fisher system
}

\author{
Fathi DKHIL and Bechir MANNOUBI
}

\begin{abstract}
This paper dressed the drift perturbation effects on the traveling wave speed in a reaction-diffusion system. We prove the existence of a traveling front solution of a KPP-Fisher equation and we show an asymptotic expansion of her speed. Finally, we discuss according all parameters of our system regions of the plane in which the traveling wave speed increases or decreases as a function of a small parameter $\varepsilon$.
\end{abstract}

\section{Introduction}

Front propagation is a phenomenon that has many scientific applications ; such as : the sprawl of epidemics and diseases, biological invasions and collective behavior, ecology, population dynamics, reaction kinetics, the flow in porous materials, etc.

This phenomenon is generally modeled by a reaction-diffusion equation of the following form

$$
\left\{\begin{array}{l}
U_{t}=\nabla \cdot(A \nabla U)+f(U) \\
U(0, x)=U_{0}(x)
\end{array}\right.
$$

where the diffusivity $A$ is a positive definite matrix and the reaction term $f$ is a $C^{2}$ nonlinear function.

Key Words: Reaction-diffusion equations, heterogeneous media, traveling wave speed, drift perturbation, KPP-Fisher equations.

2010 Mathematics Subject Classification: Primary 35B20, 35B27, 35B50; Secondary 35C20, 35K55, 35K57, 41A60.

Received: 17.02 .2014

Revised: 3.05.2014

Accepted: 12.05 .2014 . 
Understanding the mechanism of the settings of perturbation on the diffusion and (or) reaction influences the traveling fronts like configuring their location, profile, and their speed is one of the most fundamental issues. In fact, variational principles for the speed of front propagation for KPP-type nonlinearities, respectively Fisher's population genetic model as well as various other implications of the qualitative behavior of propagating waves, were given by Hadeler and Rothe in [7] and Gärtner and Freidlin in [6]. Paper [2] is used to handle the speed of traveling fronts of reactions-diffusion equations of bistable or combustion type with rapidly oscillating diffusion and drift coefficients. In the monostable case (KPP-Fisher type), the variation of the traveling wave speed was treated in [3] for nonlocally perturbed reaction-diffusion equations. Compare the survey papers $([14],[16],[15])$ in order to have an excellent reference on propagation phenomenas mathematical results especially on the existence and stability of traveling waves, and other references that has not already been cited here.

In this paper we consider a particular kind of reaction-diffusion system with drift perturbation. The type of system we are dealing with is the following

$$
\left\{\begin{array}{l}
u_{t}=\alpha \Delta u+\gamma u(1-u-v) \\
v_{t}=\beta \Delta v+\delta b \nabla v+\eta v(1-u-v)
\end{array}\right.
$$

with:

$x=\left(x_{1}, x_{2}, \ldots, x_{n}\right) \in D=\mathbb{R} \times \Omega \subset \mathbb{R}^{n}, t>0$,

$b=b\left(x_{2} \ldots x_{n}\right), \beta=\beta(\varepsilon) \longrightarrow \alpha, \eta=\eta(\varepsilon) \longrightarrow \gamma$ and $\delta=\delta(\varepsilon) \longrightarrow 0$ as $\varepsilon \longrightarrow 0$.

The cross section of the cylinder $\Omega \subset \mathbb{R}^{n-1}$ is bounded with $C^{1, \lambda}$ boundary.

For simplicity, we will consider here traveling waves in direction $e_{1}=(1,0, \ldots, 0)$. A very important question is how to study the variation of the traveling wave speed in discussing the influence of drift perturbation and all parameters $\alpha, \gamma, \beta, \delta, \eta$ of system (1).

Existence of unique monotone and stable traveling waves were shown in [5], [9], [10] and [13] for monotonic systems. A variational characterization given in [12] allows to prove an asymptotic expansion for the traveling wave speed solution of system (1).

The paper is organized as follows. In section 2, we rescale system (1) to obtain a monotone system and we show the existence of unique monotone and stable traveling wave up to translation and we give a variational characterization of the wave speed. In section 3 , we prove that the traveling wave has an asymptotic expansion. Finally in section 4, we determine regions of the plane in which the traveling wave speed solution of system (1) increases or decreases as a function of $\varepsilon$. 


\section{The variational characterization}

We rescale the system (1). So, with no loss of generality, we can suppose that $\alpha=\gamma=1$ and our system becomes

$$
\left\{\begin{array}{l}
u_{t}=\Delta u+u(1-u-v) \\
v_{t}=\beta \Delta v+\delta b \nabla v+\eta v(1-u-v)
\end{array}\right.
$$

with $b=b\left(x_{2} \ldots x_{n}\right), \beta=\beta(\varepsilon) \longrightarrow 1, \eta=\eta(\varepsilon) \longrightarrow 1$ and $\delta=\delta(\varepsilon) \longrightarrow 0$ as $\varepsilon \longrightarrow 0$.

We pose $w=1-u$. Thereby system (2) becomes

$$
\left\{\begin{aligned}
w_{t} & =\Delta w-(1-w)(w-v) \\
v_{t} & =\beta \Delta v+\delta b \nabla v+\eta v(w-v) .
\end{aligned}\right.
$$

We can easily see that system (3) is monotone. Therefore there exists a unique monotone traveling wave $\left(c_{\varepsilon}, w_{\varepsilon}, v_{\varepsilon}\right)$ in direction $e_{1}$ solution of (3) connecting two zeros of its nonlinearities; this wave is stable with respect to some subset $I_{s}$ of initial data. For more detail, see for example [5], [9], [10] and [13]. The traveling wave solution $\left(c_{\varepsilon}, w_{\varepsilon}, v_{\varepsilon}\right)$ of system (3) will satisfy the following boundary conditions

$$
\left(w_{\varepsilon}, v_{\varepsilon}\right)(-\infty)=(1,0) \quad \text { and } \quad\left(w_{\varepsilon}, v_{\varepsilon}\right)(+\infty)=\left(\frac{1}{2}, \frac{1}{2}\right) .
$$

Let $\left(c_{0}, w_{0}, v_{0}\right)$ be the traveling wave solution of the system (3) when $\varepsilon=0$ (i.e. $\beta=1, \eta=1$ and $\delta=0$ ).

Let $W_{0}=1+v_{0}-w_{0}=u_{0}+v_{0}$, then $W_{0}$ is a traveling front solution of the following KPP-Fisher equation

$$
\left\{\begin{aligned}
c_{0} W_{0}^{\prime} & =W_{0}^{\prime \prime}+W_{0}\left(1-W_{0}\right) \\
W_{0}(-\infty) & =0, W_{0}(+\infty)=1
\end{aligned}\right.
$$

with $W_{0}(t, x)=W_{0}\left(x+c_{0} t e_{1}\right)$ and $W_{0}^{\prime}$ represent the derivative of $W_{0}$ with respect to the first component $x_{1}$ of $x$.

Let

$$
W_{\varepsilon}=1+v_{\varepsilon}-w_{\varepsilon}=v_{\varepsilon}+u_{\varepsilon},
$$

then $W_{\varepsilon}$ is a traveling wave solution of the following equation

$$
\left\{\begin{array}{l}
c_{\varepsilon} W_{\varepsilon}^{\prime}=\Delta W_{\varepsilon}+W_{\varepsilon}\left(1-W_{\varepsilon}\right)+(\beta-1) \Delta v_{\varepsilon}+\delta b \nabla v_{\varepsilon}+(\eta-1) v_{\varepsilon}\left(1-W_{\varepsilon}\right) \\
W_{\varepsilon}(-\infty)=0, W_{\varepsilon}(+\infty)=1 .
\end{array}\right.
$$


Let

$$
\mathcal{K}=\left\{\begin{array}{l}
y \in C^{1}\left(\mathbb{R}, C^{2}(D)\right) \mid \partial_{t} y(t, x)>0,0<y(t, x)<1, y(0, .) \in I_{s}, \\
y(-\infty)=0, y(+\infty)=1
\end{array}\right\}
$$

be the sets of admissible comparison functions. For $y \in \mathcal{K}$ define

$$
\psi(y)=\frac{\Delta y+y(1-y)+(\beta-1) \Delta v_{\varepsilon}+\delta b \nabla v_{\varepsilon}+(\eta-1) v_{\varepsilon}(1-y)}{\partial_{t} y} .
$$

In [5] a variational characterization of the wave speed was given for more general situations. Here we will state a specific version of the result as a lemma.

LEMMA 2.1. [5] Suppose that there exists a unique stable traveling wave for problem (6) then the traveling wave speed $c_{\varepsilon}$ is given by

$$
\sup _{y \in \mathcal{K}} \inf _{(t, x) \in(\mathbb{R} \times D)} \psi(y(t, x))=c_{\varepsilon}=\inf _{y \in \mathcal{K}} \sup _{(t, x) \in(\mathbb{R} \times D)} \psi(y(t, x)) .
$$

The proof of this minimax characterization of the wave speed $c_{\varepsilon}$ is based on the maximum principle and relates technically to results given by Vol'pert et al in [12] for monotone systems of ODE's.

\section{Asymptotic expansion}

THEOREM 3.1. The traveling wave $\left(c_{\varepsilon}, w_{\varepsilon}, v_{\varepsilon}\right)$ solution of the system (3) has the following expansion:

$$
\begin{aligned}
c_{\varepsilon} & =c_{0}+\varepsilon c_{1}+o(\varepsilon) \\
v_{\varepsilon} & =v_{0}+\varepsilon v_{1}+o(\varepsilon) \\
w_{\varepsilon} & =w_{0}+\varepsilon w_{1}+o(\varepsilon) .
\end{aligned}
$$

Proof. We note $\beta^{\prime}=\left.\frac{d \beta}{d \varepsilon}\right|_{\varepsilon=0}, \eta^{\prime}=\left.\frac{d \eta}{d \varepsilon}\right|_{\varepsilon=0}$ and $\delta^{\prime}=\left.\frac{d \delta}{d \varepsilon}\right|_{\varepsilon=0}$ then equation (6) becomes

$$
W_{\varepsilon t}=\Delta W_{\varepsilon}+W_{\varepsilon}\left(1-W_{\varepsilon}\right)+\varepsilon\left[\beta^{\prime} \Delta v_{\varepsilon}+\delta^{\prime} b \nabla v_{\varepsilon}+\eta^{\prime} v_{\varepsilon}\left(1-W_{\varepsilon}\right)\right]+o(\varepsilon) .
$$

Since $W_{\varepsilon}$ and $v_{\varepsilon}$ are regular and bounded functions, then the comparison principle implies that

$$
W_{\varepsilon}=W_{0}+\varepsilon W_{1}+o(\varepsilon) .
$$


Furthermore, we have

$$
\left\{\begin{array}{l}
u_{\varepsilon t}=\Delta u_{\varepsilon}+u_{\varepsilon}\left(1-u_{\varepsilon}-v_{\varepsilon}\right) \\
u_{0 t}=\Delta u_{0}+u_{0}\left(1-u_{0}-v_{0}\right)
\end{array}\right.
$$

We substract these two equations to get

$$
\left(u_{\varepsilon}-u_{0}\right)_{t}-\Delta\left(u_{\varepsilon}-u_{0}\right)-\left(u_{\varepsilon}-u_{0}\right)\left(1-u_{0}-v_{0}\right)=u\left(W_{0}-W_{\varepsilon}\right) .
$$

Using the comparison principle, we obtain

$$
u_{\varepsilon}=u_{0}+\varepsilon u_{1}+o(\varepsilon) .
$$

Therefore, we deduce from (5) that

$$
w_{\varepsilon}=w_{0}+\varepsilon w_{1}+o(\varepsilon)
$$

and

$$
v_{\varepsilon}=v_{0}+\varepsilon v_{1}+o(\varepsilon)
$$

which justify our expansion for $v_{\varepsilon}$ and $w_{\varepsilon}$.

To show the asymptotic expansion of $c_{\varepsilon}$, we consider the test function:

$$
y(x)=W_{0}+\varepsilon y_{1}(x)
$$

where $y_{1}$ will be determined below.

An easy computation gives that

$$
\begin{aligned}
\psi(y)= & \frac{\Delta y+y(1-y)+(\beta-1) \Delta v_{\varepsilon}+\delta b \nabla v_{\varepsilon}+(\eta-1) v_{\varepsilon}(1-y)}{\partial_{t} y} \\
= & \frac{c_{0} W_{0}^{\prime}+\varepsilon\left[y_{1}^{\prime \prime}+\left(1-2 W_{0}\right) y_{1}+\eta^{\prime} v_{0}\left(1-W_{0}\right)+\beta^{\prime} v_{0}^{\prime \prime}+\delta^{\prime} b v_{0}^{\prime}\right]}{W_{0}^{\prime}+\varepsilon y_{1}^{\prime}}+o(\varepsilon) \\
= & c_{0}+\frac{\varepsilon}{W_{0}^{\prime}}\left[y_{1}^{\prime \prime}-c_{0} y_{1}^{\prime}+\left(1-2 W_{0}\right) y_{1}+\eta^{\prime} v_{0}\left(1-W_{0}\right)+\beta^{\prime} v_{0}^{\prime \prime}+\delta^{\prime} b v_{0}^{\prime}\right] \\
& +o(\varepsilon) .
\end{aligned}
$$

We choose $y_{1}$ such that the coefficient of $\varepsilon$ in $\psi(y)$ is constant. Thus $y_{1}$ solves the following equation

$$
\left\{\begin{aligned}
y_{1}^{\prime \prime}-c_{0} y_{1}^{\prime}+\left(1-2 W_{0}\right) y_{1} & =-c_{1} W_{0}^{\prime}-\eta^{\prime} v_{0}\left(1-W_{0}\right)-\beta^{\prime} v_{0}^{\prime \prime}-\delta^{\prime} b v_{0}^{\prime} \\
y_{1}( \pm \infty) & =0 .
\end{aligned}\right.
$$


Near $\pm \infty$ all derivatives of $W_{0}$ have the same exponential decay rate as $W_{0}^{\prime}$. Therefore with our choice of $y$ we have $\partial_{t} y>0$ for small $\varepsilon$. Thus $y$ is an admissible function for the minimax characterization (7) and we have

$$
c_{\varepsilon}=c_{0}+\varepsilon c_{1}+o(\varepsilon)
$$

This ends the proof of Theorem 3.1.

\section{Sign of $c_{1}$}

In this section, we discuss according to derivative perturbations parameters how $c_{1}$ may change sign.

PROPOSITION 4.1. Let $\left(c_{\varepsilon}, w_{\varepsilon}, v_{\varepsilon}\right)$ be the traveling wave solution of system (3), then the variation of $c_{\varepsilon}$ is as follows.

- If $c_{0} \beta^{\prime}+\delta^{\prime} \oint b \leq 0$ and $\eta^{\prime}-\beta^{\prime} \leq-\frac{c_{0}+\sqrt{c_{0}^{2}-4}}{2}\left(c_{0} \beta^{\prime}+\delta^{\prime} \oint b\right)$

then $c_{\varepsilon}$ decreases as a function of $\varepsilon$.

- If $c_{0} \beta^{\prime}+\delta^{\prime} \oint b \geq 0$ and $\eta^{\prime}-\beta^{\prime} \geq-\frac{c_{0}+\sqrt{c_{0}^{2}-4}}{2}\left(c_{0} \beta^{\prime}+\delta^{\prime} \oint b\right)$

then $c_{\varepsilon}$ increases as a function of $\varepsilon$.

Proof. We note $w^{\prime}$ and $v^{\prime}$ the derivatives of $w$ and $v$ with respect to the first variable then we have

$$
\left\{\begin{array}{l}
c_{\varepsilon} w_{\varepsilon}^{\prime}=\Delta w_{\varepsilon}-\left(1-w_{\varepsilon}\right)\left(w_{\varepsilon}-v_{\varepsilon}\right) \\
c_{\varepsilon} v_{\varepsilon}^{\prime}=\beta \Delta v_{\varepsilon}+\delta b v_{\varepsilon}^{\prime}+\eta v_{\varepsilon}\left(w_{\varepsilon}-v_{\varepsilon}\right)
\end{array}\right.
$$

this can be writen as

$$
\left\{\begin{aligned}
\left(c_{0}+\varepsilon c_{1}\right)\left(w_{0}+\varepsilon w_{1}\right)^{\prime}= & \Delta\left(w_{0}+\varepsilon w_{1}\right)-\left(1-w_{0}-\varepsilon w_{1}\right)\left(w_{0}+\varepsilon w_{1}-v_{0}\right. \\
& \left.-\varepsilon v_{1}\right)+o(\varepsilon) \\
\left(c_{0}+\varepsilon c_{1}\right)\left(v_{0}+\varepsilon v_{1}\right)^{\prime}= & \beta \Delta\left(v_{0}+\varepsilon v_{1}\right)+\delta b\left(v_{0}+\varepsilon v_{1}\right)^{\prime} \\
& +\eta\left(v_{0}+\varepsilon v_{1}\right)\left(w_{0}+\varepsilon w_{1}-v_{0}-\varepsilon v_{1}\right)+o(\varepsilon) .
\end{aligned}\right.
$$


Since $\left(c_{0}, w_{0}, v_{0}\right)$ is a solution of the system (3) when $\varepsilon=0$ then we have

$$
\left\{\begin{aligned}
c_{1} w_{0}^{\prime}+c_{0} w_{1}^{\prime}= & \Delta w_{1}-\left(1-w_{0}\right)\left(w_{1}-v_{1}\right)+w_{1}\left(w_{0}-v_{0}\right) \\
c_{1} v_{0}^{\prime}+c_{0} v_{1}^{\prime}= & \beta^{\prime} \Delta v_{0}+\Delta v_{1}+\eta^{\prime} v_{0}\left(w_{0}-v_{0}\right)+\delta^{\prime} b v_{0}^{\prime}+v_{0}\left(w_{1}-v_{1}\right) \\
& +v_{1}\left(w_{0}-v_{0}\right)
\end{aligned}\right.
$$

which implies that

$$
\left\{\begin{aligned}
& \Delta w_{1}-c_{0} w_{1}^{\prime}-\left(1-w_{0}\right)\left(w_{1}-v_{1}\right)+w_{1}\left(w_{0}-v_{0}\right)=c_{1} w_{0}^{\prime} \\
& \Delta v_{1}-c_{0} v_{1}^{\prime}+v_{0}\left(w_{1}-v_{1}\right)+v_{1}\left(w_{0}-v_{0}\right)= c_{1} v_{0}^{\prime}-\beta^{\prime} \Delta v_{0}-\eta^{\prime} v_{0}\left(w_{0}-v_{0}\right) \\
&-\delta^{\prime} b v_{0}^{\prime} .
\end{aligned}\right.
$$

We substract these two equations to obtain

$$
\begin{gathered}
\Delta\left(v_{1}-w_{1}\right)-c_{0}\left(v_{1}-w_{1}\right)^{\prime}-\left(v_{1}-w_{1}\right)\left(1+2\left(v_{0}-w_{0}\right)\right) \\
=c_{1}\left(v_{0}-w_{0}\right)^{\prime}-\beta^{\prime} \Delta v_{0}-\eta^{\prime} v_{0}\left(w_{0}-v_{0}\right)-\delta^{\prime} b v_{0}^{\prime} .
\end{gathered}
$$

We pose $\phi=v_{1}-w_{1}$ then this equation is equivalent to

$$
\Delta \phi-c_{0} \phi^{\prime}-\phi\left(1-2 W_{0}\right)=c_{1} W_{0}^{\prime}-\beta^{\prime} \Delta v_{0}-\eta^{\prime} v_{0}\left(w_{0}-v_{0}\right)-\delta^{\prime} b v_{0}^{\prime} .
$$

Using that $W_{0}$ is a solution of (4) and the Fredholm alternative, the solvability condition of the equation (9) can be writen as

$$
\begin{aligned}
c_{1} \int_{\mathbb{R} \times \Omega} W_{0}^{\prime 2} e^{-c_{0} \xi}= & \beta^{\prime} \int_{\mathbb{R} \times \Omega} \Delta v_{0} W_{0}^{\prime} e^{-c_{0} \xi} d x+\eta^{\prime} \int_{\mathbb{R} \times \Omega} v_{0}\left(1-W_{0}\right) W_{0}^{\prime} e^{-c_{0} \xi} \\
& +\delta^{\prime} \int_{\mathbb{R} \times \Omega} b v_{0}^{\prime} W_{0}^{\prime} e^{-c_{0} \xi} .
\end{aligned}
$$

Since $v_{0}$ satisfies $\Delta v_{0}=c_{0} v_{0}^{\prime}-v_{0}\left(1-W_{0}\right)$ and using the fuct that $v_{0}$ and $W_{0}$ are independent on $\left(x_{2} . . x_{n}\right) \in \Omega$ then the solvability condition is equivalent to

$$
\begin{aligned}
c_{1} \int_{\mathbb{R}} W_{0}^{\prime 2} e^{-c_{0} \xi} & =c_{0} \beta^{\prime} \int_{\mathbb{R}} v_{0}^{\prime} W_{0}^{\prime} e^{-c_{0} \xi}+\left(\eta^{\prime}-\beta^{\prime}\right) \int_{\mathbb{R}} v_{0}\left(1-W_{0}\right) W_{0}^{\prime} e^{-c_{0} \xi} \\
& +\delta^{\prime} \oint b \int_{\mathbb{R}} v_{0}^{\prime} W_{0}^{\prime} e^{-c_{0} \xi} .
\end{aligned}
$$

After an integration by part we obtain that

$$
c_{1} \int_{\mathbb{R}}{W_{0}^{\prime 2}}^{2} e^{-c_{0} \xi}=\int_{\mathbb{R}}\left(\left(c_{0} \beta^{\prime}+\delta^{\prime} \oint b\right) W_{0}+\left(\eta^{\prime}-\beta^{\prime}\right) W_{0}^{\prime}\right) v_{0}\left(1-W_{0}\right) e^{-c_{0} \xi} .
$$

We know that $v_{0}\left(1-W_{0}\right)>0$, so that if $\left(c_{0} \beta^{\prime}+\delta^{\prime} \oint b\right) W_{0}+\left(\eta^{\prime}-\beta^{\prime}\right) W_{0}^{\prime}$ does not change sign then we can deduce the sign of $c_{1}$. Here we have three cases. 
First case: $\left(c_{0} \beta^{\prime}+\delta^{\prime} \oint b\right)$ and $\left(\eta^{\prime}-\beta^{\prime}\right)$ have the same sign then it is the sign of $c_{1}$.

Second case: one of $\left(c_{0} \beta^{\prime}+\delta^{\prime} \oint b\right)$ and $\left(\eta^{\prime}-\beta^{\prime}\right)$ is zero then the sign of $c_{1}$ is the sign of the non zero term.

Third case: $\left(c_{0} \beta^{\prime}+\delta^{\prime} \oint b\right)$ and $\left(\eta^{\prime}-\beta^{\prime}\right)$ have an opposite sign. In this case we need a good estimate on $W_{0}^{\prime}$.

Equation (4) is equivalent to

$$
W_{0}^{\prime \prime}-\left(c_{0}-a\right) W_{0}^{\prime}=a W_{0}^{\prime}-W_{0}\left(1-W_{0}\right) \quad \forall a \in \mathbb{R} .
$$

Since $0<W_{0}<1$ then we have

$$
W_{0}^{\prime \prime}-\left(c_{0}-a\right) W_{0}^{\prime}>a\left(W_{0}^{\prime}-\frac{1}{a} W_{0}\right) \quad \forall a \neq 0 .
$$

We choose $a$ such that $c_{0}-a=\frac{1}{a}$ which is possible since we have $c_{0}^{2} \geq 4$, (see [1] and [11]).

We note

$$
a_{ \pm}=\frac{c_{0} \pm \sqrt{c_{0}^{2}-4}}{2}>0 .
$$

After multiplication by $e^{-\frac{1}{a} \xi}$ and integrating the inequality (10) on $(\xi, \infty)$ for any $\xi \in \mathbb{R}$ we obtain that

$$
W_{0}^{\prime}<a W_{0}
$$

with $a=a_{-}=\frac{c_{0}-\sqrt{c_{0}^{2}-4}}{2}$.

We discuss two subcases

1. $\eta^{\prime}-\beta^{\prime}>0$ and $c_{0} \beta^{\prime}+\delta^{\prime} \oint b<0$. We have that

$$
\begin{aligned}
\left(c_{0} \beta^{\prime}+\delta^{\prime} \oint b\right) W_{0}+\left(\eta^{\prime}\right. & \left.-\beta^{\prime}\right) W_{0}^{\prime}<\left(c_{0} \beta^{\prime}+\delta^{\prime} \oint b+a_{-}\left(\eta^{\prime}-\beta^{\prime}\right)\right) W_{0} \\
& <\left(\frac{c_{0}}{2}\left(\eta^{\prime}+\beta^{\prime}\right)+\delta^{\prime} \oint b-\frac{\eta^{\prime}-\beta^{\prime}}{2} \sqrt{c_{0}^{2}-4}\right) W_{0} .
\end{aligned}
$$


So that if $\frac{c_{0}}{2}\left(\eta^{\prime}+\beta^{\prime}\right)+\delta^{\prime} \oint b<0$ then $c_{1}<0$.

If not, we have

$$
\frac{c_{0}}{2}\left(\eta^{\prime}+\beta^{\prime}\right)+\delta^{\prime} \oint b>0,
$$

therefore an easy computation gives that

$$
\frac{c_{0}}{2}\left(\eta^{\prime}+\beta^{\prime}\right)+\delta^{\prime} \oint b-\frac{\eta^{\prime}-\beta^{\prime}}{2} \sqrt{c_{0}^{2}-4} \leq 0,
$$

if and only if

$$
\frac{\left(-c_{0}+\sqrt{c_{0}^{2}-4}\right)}{2}\left(c_{0} \beta^{\prime}+\delta^{\prime} \oint b\right) \leq \eta^{\prime}-\beta^{\prime}
$$

and

$$
\eta^{\prime}-\beta^{\prime} \leq-\frac{\left(c_{0}+\sqrt{c_{0}^{2}-4}\right)}{2}\left(c_{0} \beta^{\prime}+\delta^{\prime} \oint b\right) .
$$

Hence, in this subcase we have $c_{1}<0$ and then $c_{\varepsilon}$ decreases as a function of $\varepsilon$, for $\varepsilon$ small enough.

2. $\eta^{\prime}-\beta^{\prime}<0$ and $c_{0} \beta^{\prime}+\delta^{\prime} \oint b>0$. We have that

$$
\begin{array}{r}
\left(c_{0} \beta^{\prime}+\delta^{\prime} \oint b\right) W_{0}+\left(\eta^{\prime}-\beta^{\prime}\right) W_{0}^{\prime}>\left(c_{0} \beta^{\prime}+\delta^{\prime} \oint b+a_{-}\left(\eta^{\prime}-\beta^{\prime}\right)\right) W_{0} \\
>\left(\frac{c_{0}}{2}\left(\eta^{\prime}+\beta^{\prime}\right)+\delta^{\prime} \oint b-\frac{\eta^{\prime}-\beta^{\prime}}{2} \sqrt{c_{0}^{2}-4}\right) W_{0} .
\end{array}
$$

So that if $\frac{c_{0}}{2}\left(\eta^{\prime}+\beta^{\prime}\right)+\delta^{\prime} \oint b>0$ then $c_{1}>0$.

If not, we have

$$
\frac{c_{0}}{2}\left(\eta^{\prime}+\beta^{\prime}\right)+\delta^{\prime} \oint b<0,
$$

therefore an easy computation gives that

$$
\frac{c_{0}}{2}\left(\eta^{\prime}+\beta^{\prime}\right)+\delta^{\prime} \oint b-\frac{\eta^{\prime}-\beta^{\prime}}{2} \sqrt{c_{0}^{2}-4} \geq 0,
$$

if and only if

$$
-\frac{c_{0}+\sqrt{c_{0}^{2}-4}}{2}\left(c_{0} \beta^{\prime}+\delta^{\prime} \oint b\right) \leq \eta^{\prime}-\beta^{\prime}
$$


and

$$
\eta^{\prime}-\beta^{\prime} \leq \frac{-c_{0}+\sqrt{c_{0}^{2}-4}}{2}\left(c_{0} \beta^{\prime}+\delta^{\prime} \oint b\right)
$$

Hence, in this subcase we have $c_{1}>0$ and then $c_{\varepsilon}$ increases as a function of $\varepsilon$, for $\varepsilon$ small enough.

This ends the proof of proposition 4.1.

Return now to the full system (1). A simple scaling argument allow to conclude the result in a general way. We note $c_{\varepsilon}^{*}$ the traveling wave speed solution of (1). Then we have the following

THEOREM 4.2. Let $\left(c_{\varepsilon}^{*}, u_{\varepsilon}^{*}, v_{\varepsilon}^{*}\right)$ be the traveling wave solution of system (1) for $\varepsilon$ small enough. Then $c_{\varepsilon}^{*}=\frac{c_{\varepsilon}}{\alpha}$ and has the following variations.

- If $\quad \frac{c_{0}}{\alpha} \beta^{\prime}+\delta^{\prime} \oint b \leq 0 \quad$ and $\quad \alpha \eta^{\prime}-\gamma \beta^{\prime} \leq-\frac{c_{0}+\sqrt{c_{0}^{2}-4 \alpha \gamma}}{2}\left(\frac{c_{0}}{\alpha} \beta^{\prime}+\delta^{\prime} \oint b\right)$ then $c_{\varepsilon}^{*}$ decreases as a function of $\varepsilon$.

- If $\quad \frac{c_{0}}{\alpha} \beta^{\prime}+\delta^{\prime} \oint b \geq 0 \quad$ and $\quad \alpha \eta^{\prime}-\gamma \beta^{\prime} \geq-\frac{c_{0}+\sqrt{c_{0}^{2}-4 \alpha \gamma}}{2}\left(\frac{c_{0}}{\alpha} \beta^{\prime}+\delta^{\prime} \oint b\right)$ then $c_{\varepsilon}^{*}$ increases as a function of $\varepsilon$.

Where $c_{\varepsilon}$ is the traveling wave speed solution of (3).

Figure 1 presents the different regions of the plane

$$
\left(X=\frac{c_{0}}{\alpha} \beta^{\prime}+\delta^{\prime} \oint b, \quad Y=\alpha \eta^{\prime}-\gamma \beta^{\prime}\right)
$$

in which $c_{\varepsilon}^{*}$ increases or decreases as a function of $\varepsilon$.

REMARK 4.3. In the regions

$$
\left(X>0, Y<-\frac{c_{0}+\sqrt{c_{0}^{2}-4 \alpha \gamma}}{2} X\right) \text { and }\left(X<0, Y>-\frac{c_{0}+\sqrt{c_{0}^{2}-4 \alpha \gamma}}{2} X\right)
$$

of Figure 1, the determination of the sign of $c_{1}$ rest an open problem. 


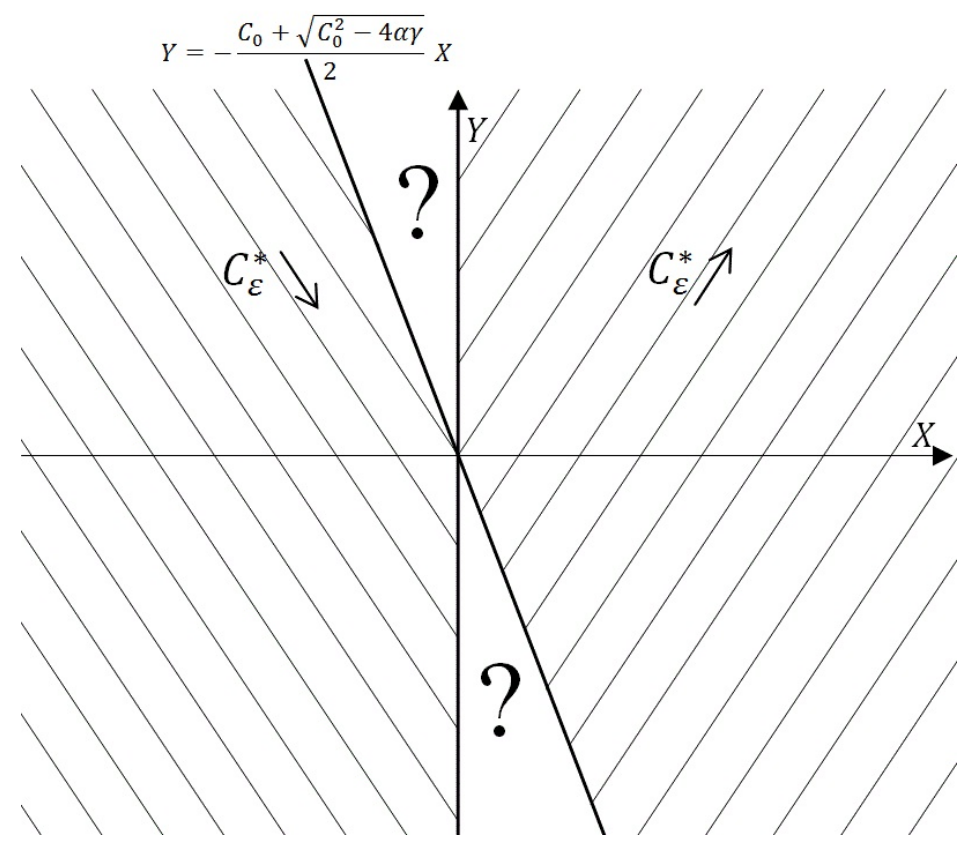

Figure 1: $c_{\varepsilon}^{*}$ variation.

\section{Acknowledgment}

The authors are very grateful to Hatem Zaag for his helpful comments.

\section{References}

[1] D.G. Anderson, H.F. Weinberg, "multidimensional nonlinear diffusions arising in population genetics," Adv.Math.30:(7978), 33-76.

[2] F. Dkhil, A. Stevens, "Traveling wave speeds in rapidly oscillating media," Discrete and continuous dynamical system 25, no.1 (2009), 89-108.

[3] F. Dkhil, A. Stevens, "Traveling wave speeds of nonlocally perturbed reactiondiffusion equations," Asymptotic Analysis 46, (2006), 81-91.

[4] S. Heinze, "Wave solutions to reaction-diffusion systems in perfored domains," Z. Anal. Anwendungen, 20, no.3, (2001), 661-676.

[5] S. Heinze, G. Papanicolaou, A. Stevens, "Variational principle for propagation speeds in inhomogeneous media," SIAM J. Appl. Math. vol. 62, no.1, (2001), 129-148. 
[6] J. Gärtner, M.I. Freidlin, "On the propagation of concentration waves in periodic and random media," Soviet. Math. Dokl., 20, (1979), 1282-1286.

[7] K.P. Hadeler, F. Rothe, "Traveling fronts in nonlinear diffusion equations," J. Math. Biol. 2, (1975), 251-263.

[8] F. Hamel, "Folmules min-max pour les vitesse d'ondes progressive multidimensionelles," Ann. Fac. Sci. Toulouse Math. (6), 8, (1999), 259-280.

[9] X. Hou, "On the minimal speed and asymptotics of the wave solutions for the lotka volterra system," Analysis of PDEs (math.AP); Dynamical Systems (math.DS) (2010).

[10] X. Hou, W. Feng, "Traveling waves and their Stability for a Public Goods Game Model," Analysis of PDEs (math.AP) (2009).

[11] A.N. Kolmogorov, I.G. Petrovsky, N.S. Pisknnov "Etude de l'quation de la diffusion avec croissance de la quantit de matire et son application un problme biologique," Bulletin Universit d'Etat Moskou (Bjul.Moskourskogo Gos.Univ), (1937), 1-26.

[12] A.I. Vol'pert, V. Vol'pert, V.A. Vol'pert, "Traveling wave solutions of parabolic systems," Translations of Mathematical Monographs, 140, Providence, RI: American Mathematical Society, (1994).

[13] A. W Leung, X. Hou, W. Feng, "Traveling Wave Solutions for LotkaVolterra System Re-Visited," Analysis of PDEs (math.AP); Spectral Theory (math.SP) (2009).

[14] J. Xin, "Front propagation in heterogeneous media." SIAM Rev., 42, (2000), 161-230.

[15] J. Xin, "Existence of planar flame fronts in convective-diffusive periodic media," Arch. Ration. Mech. Anal., 121, (1992), 205-233.

[16] J. Xin, "Existence and stability of traveling waves in periodic media governed by a bistable nonlinearity," J. Dynam. Differential Equations, 3, (1991), 541-573.

Fathi DKHIL,

Département de Mathématiques,

Institut Supérieur d'Informatique,

Université Tunis El-Manar,

2 rue Abou Raihan Bayrouni, 2080 Ariana, Tunisia.

Email: fathi.dkhil@gmail.com

Bechir MANNOUBI

Département de Mathématiques,

Institut Supérieur d'Informatique,

Université Tunis El-Manar,

2 rue Abou Raihan Bayrouni, 2080 Ariana, Tunisia.

Email: bechir_ca@live.com 